\title{
Evaluation of a New Enzyme-Linked Immunosorbent Assay (ELISA) for Johne's Disease in Goat Serum
}

\author{
Ron White $^{1 *}$, Keith R. Marotti ${ }^{1}$, Murray E. Hines ${ }^{2}$ II, Lisa Whittington ${ }^{2}$, Kristie Goins ${ }^{2}$, Chinta \\ Lamichhane $^{3}$, Dan Lin ${ }^{4}$, Ann Robinson ${ }^{5}$ \\ ${ }^{1}$ Zoetis, Global Diagnostics, Veterinary Medicine Research and Development, 333 Portage Street, Kalamazoo, MI 49007, Michigan, United States \\ ${ }^{2}$ The University of Georgia, College of Veterinary Medicine, Tifton Veterinary Diagnostic and Investigational Laboratory, P.O. Box 1389, 43 Brighton Road, Tifton, GA \\ 31793-1389, Georgia, United States \\ ${ }^{3}$ Zoetis, Global Diagnostics, Veterinary Medicine Research and Development, 8075 Greenmead Drive, College Park, MD 20742, Maryland, United States \\ ${ }^{4}$ Zoetis, Biometrics, Veterinary Medicine Research and Development, Zaventem, Belgium \\ ${ }^{5}$ Chef du Monde SL, Animal Health Language Services, Calle Sepúlveda 143, Ático 3, 08011 Barcelona, Spain
}

Received: December 14, 2015; Accepted: February 06, 2016; Published: February 12, 2016

*Corresponding author: Ron White, Zoetis, Global Diagnostics, Veterinary Medicine, Research and Development, 333 Portage St, Kalamazoo, Michigan, United States. E-mail: ron.white@zoetis.com

\begin{abstract}
Control of Johne's Disease (JD) is a high priority, it causes significant economic losses in the livestock industry. An efficacious vaccine for JD is not currently available and no satisfactory treatment exists, so control programs depend on diagnostic tests based on detection of the immune response generated after infection with Mycobacterium avium subsp. paratuberculosis (MAP)-the causative agent of JD-to identify and remove infected animals and management practices to interrupt transmission.

Objectives: The objective of this study was to evaluate a new, commercially-available, Enzyme-Linked, Immunosorbent Assay (ELISA) that was developed for cattle, for use with goat serum. For comparison, a United States Department of Agriculture (USDA)-approved ELISA kit for goats, an Agar Gel Immunodiffusion (AGID) test and a Polymerase Chain Reaction (PCR) test to detect MAP in fecal samples were also evaluated.

Methods: Infection status at necropsy was taken as the reference standard, determined by gross, histological and microbiological examination of tissue specimens. The serum and fecal samples were taken from goat kids that had participated in a previous vaccine efficacy study. Eighty goat kids were vaccinated either once subcutaneously at eight weeks with a commercial vaccine, or orally at eight and 10 weeks with one of five experimental oral vaccines or a sham-control oral vaccine. Kids were challenged orally with a bovine isolate of MAP, three weeks after the last vaccination.

Results: None of the vaccines prevented infection. Samples taken from 10 to 13 months post-challenge were used to compare the assays. The specificity of all tests was 100\%. At 13 months, the new ELISA, approved ELISA, AGID test and fecal PCR test had sensitivities of 55.7\%, 52.9\%, $24.3 \%$ and $87.1 \%$ respectively. At 11 months the sensitivities were $48.6 \%, 38.6 \%, 10.0 \%$ and $78.6 \%$ respectively and the sensitivity of the new ELISA was significantly better than the approved ELISA $(p=0.0196)$.

Conclusion: The new ELISA effectively detected circulating MAP antibody in the serum of infected goats and the preliminary results in this study indicate the kit has potential for use in goat JD control programs. The results for the new ELISA in goat serum are comparable with those reported when the ELISA test is used for cattle serum.
\end{abstract}

Keywords: Mycobacterium avium subspecies paratuberculosis; MAP; Comparison; Goats; Serology; ELISA; Johne’s disease

\section{Introduction}

Johne's disease (JD) is a chronic, progressive, intestinal disease caused by Mycobacterium avium subspecies paratuberculosis (MAP). It has a worldwide distribution, and a variety of animal species can be infected, but the primary hosts are ruminants, including goats.

The primary route of transmission in all species is thought to be fecal-oral $[1,2]$. MAP is excreted in the feces of infected animals $[1,2]$ and has been isolated from milk and colostrum from infected cattle. Young cattle and sheep can easily be infected by experimental oral transmission [1]. Age-related resistance to infection has been demonstrated for cattle [2] and is assumed to be the case for all ruminants. Cross species transmission is possible, including in wildlife. MAP is highly resistant in the environment [3].

There are four phases of the disease. First, silent infection, which can be detected only by necropsy and tissue culture. Second, subclinical disease, in which only a small percentage of animals have detectable antibodies and most animals are fecalculture-negative with intermittent shedding of low numbers of MAP in feces in only a few animals. Third, clinical disease, often seen several years after infection. In cattle, clinical signs are of gradual weight loss, rough hair coat, and diarrhea and drop in 
milk production. In goats, signs are similar, but onset occurs in younger animals and diarrhea is less common and, when present, is intermittent, although it can be severe in some goats in individual herds $[2,4]$. Fourth and final, advanced clinical disease (end-stage). Once clinical signs are apparent, most animals will have detectable antibodies and MAP can be detected in feces.

An efficacious vaccine for JD is not currently available and no satisfactory treatment exists, so control programs depend upon testing to identify and remove infected animals and management practices to interrupt transmission of MAP [5]. Control of JD is a high priority, because it causes significant economic losses in the livestock industry [6,7]. In addition, there is a potential, as yet unproven, link with Crohn's Disease in humans. Viable MAP has been demonstrated in a variety of meat and dairy products, confirming that humans are continuously exposed to MAP [8]. This could increase pressure on producers to implement testing and control programs in the future.

Diagnosis of JD is difficult, because clinical signs of diarrhea and loss of body condition are similar to many other common ruminant diseases. During the very long subclinical phase of JD, infected animals spread infection, but do not show clinical signs and are difficult to detect using diagnostic tests, because shedding of MAP occurs at a very low level and is intermittent in this phase [9]. As a result, testing at herd-level rather than individual-level is recommended.

A valuable screening test for JD control programs is the Enzyme-Linked Immunosorbent Assay (ELISA) for antibodies against MAP. The test has relatively low sensitivity in individual animals in the subclinical phase (for the reasons mentioned above), but has fairly good sensitivity at the herd level and relatively high sensitivity during the clinical phase. It can be performed using milk or serum samples, is relatively low cost, simple to perform and gives rapid results. For these reasons, ELISA-testing is preferred over fecal culture or PCR in most herd situations [9].

A new, improved, commercially-available, ELISA to detect MAP-antibody has been developed for both serum- and milk testing in cattle. The purpose of the current study was to evaluate this new ELISA for its utility in goats to determine if the improvement noted in cattle samples also held true in goat serum samples. It was directly compared with two other serological assays. The serum and fecal samples were obtained from goats that were experimentally-infected with MAP during a vaccine efficacy study to compare experimental JD vaccine candidates [10]. The sensitivity and specificity of all the tests were determined by comparison with confirmed infection status at necropsy, based on scoring severity of gross and microscopic lesions, relative numbers of Acid-Fast Bacilli (AFB), and detection of MAP by culture or PCR.

\section{Methods}

\section{Experimental samples from goat kids}

The serum and fecal samples used in the study were collected from goats that had participated in a recent JD randomized vaccine efficacy study [10]. Eighty goat kids were vaccinated once subcutaneously at eight weeks of age with a commercial vaccine (SILIRUM $^{\circledR}$, Zoetis, New Jersey, United States) or orally, twice, at eight and 10 weeks of age with one of five oral experimental vaccines or an oral sham-control vaccine consisting of pasteurized goat's milk. Three weeks after the last vaccination, the kids were challenged orally with a total of $200 \mathrm{mg}$ pelleted wet weight MAP (totaling $1.44 \times 10^{9} \mathrm{CFU}$ ), divided into two consecutive daily 100 mg doses (approximately $.72 \times 10^{9} \mathrm{CFU}$ each) using bovine MAP isolate ATCC-700535 (K10-like strain). There were eight groups in total, each containing 10 kids [10].

Kids within each group were euthanized and necropsied at 13 months post-challenge. Infection status at necropsy was determined by evaluating and scoring, gross and microscopic lesions, relative number of AFB, and detection of MAP by culture or PCR. The lesion scores were summed to give a final necropsy lesion score. Lesion scores indicated that all challenged kids had signs compatible with JD, suggesting that none of the vaccines prevented infection. All kids in the negative control group (Group 1 , non-challenged) had final lesion scores of 0 and negative PCR and cultures, confirming that they were not infected with MAP [10]. Table 1 list the groups, treatments, and infection status at necropsy. Blood- and fecal samples were collected prior to vaccination (baseline samples) and at monthly intervals throughout the study [10]. Serum and fecal samples taken from 10 to 13 months post-challenge were used to compare all the assays in the study. The new ELISA (SERELISA ${ }^{\circledR}$ ParaTB ELISA kit. Zoetis, New Jersey, United States) was compared to the approved ELISA kit for goats $\left(\right.$ ParaCHEK $^{\circledR}$, Biocor Animal Health, Nebraska, United States), an Agar Gel Immunodiffusion (AGID) test using serum samples and to a Polymerase Chain Reaction (PCR) test to detect MAP in fecal samples. The confirmed infection status at necropsy was used as the reference status for comparison for all tests. The study was conducted one time on each of the four tests.

Table 1: Summary of experimental groups in the vaccine study [11].

\begin{tabular}{|l|l|l|l|}
\hline $\begin{array}{l}\text { Experimental } \\
\text { Group }\end{array}$ & Description & Treatment & $\begin{array}{l}\text { MAP Infection } \\
\text { Status at } \\
\text { Necropsy }\end{array}$ \\
\hline Group 1 & Negative control & $\begin{array}{l}\text { Sham-vaccine, } \\
\text { non-challenged }\end{array}$ & Negative \\
\hline Group 2 & Commercial Vaccine & $\begin{array}{l}\text { Vaccinated, } \\
\text { challenged }\end{array}$ & Positive \\
\hline Group 3 & 316 Vaccine & $\begin{array}{l}\text { Vaccinated, } \\
\text { challenged }\end{array}$ & Positive \\
\hline Group 4 & 315 Vaccine & $\begin{array}{l}\text { Vaccinated, } \\
\text { challenged }\end{array}$ & Positive \\
\hline Group 5 & 319 Vaccine & $\begin{array}{l}\text { Vaccinated, } \\
\text { challenged }\end{array}$ & Positive \\
\hline Group 6 & 318 Vaccine & $\begin{array}{l}\text { Vaccinated, } \\
\text { challenged }\end{array}$ & Positive \\
\hline Group 7 & 329 Vaccine & $\begin{array}{l}\text { Vaccinated, } \\
\text { challenged }\end{array}$ & Positive \\
\hline Group 8 & Positive control & $\begin{array}{l}\text { Sham-vaccine, } \\
\text { challenged }\end{array}$ & Positive \\
\hline
\end{tabular}

Each group contained 10 kids 


\section{Serological Assays}

The new ELISA was performed according to the manufacturer's instructions. The approved ELISA was performed according to the manufacturer's recommendations. For both ELISA tests the cut-off threshold was set at an ELISA optical density (S/P ratio) of 0.7. The principle of the SERELISA test is as follows: Serum obtained from bovine herds exposed to ParaTB antigens contains specific ParaTB antibodies. Serum, diluted in sample diluent, is added to antigen coated wells. Specific antibody in the serum forms an antibody-antigen complex with the antigen bound to the plate. After washing the plate, a monoclonal anti-bovine IgG HRP peroxidase conjugate is added to each well. The antibodyantigen complex remaining from the previous step binds with the conjugate. After a brief incubation period, the unbound conjugate is removed by a second wash step. Substrate, which contains a chromagen (ABTS), is added to each well. Chromagen color change (from clear to green-blue) occurs in the presence of the peroxidase enzyme. The relative intensity of color developed in 15 minutes (compared to controls) is directly proportional to the level of antibody in the serum. After the substrate has incubated Stop Solution is added to each well to terminate the reaction and the plate is read using an ELISA plate reader at $405-410 \mathrm{~nm}$.

Reagents and methods of the Johne's AGID protocol at the New York Animal Health Diagnostic Laboratory (NYAHDL) were used for AGID testing [10,11]. AGID was performed in $0.8 \%$ agarose gel prepared in phosphate buffered saline on petri plates $(100 \mathrm{~mm} \times 15 \mathrm{~mm})$ using an immunodiffusion template to create a seven-well pattern, with a center well and six outside wells. The number of well patterns per plate depended upon the number of samples to be tested. The test was performed using protoplasmic antigen (Protoplasmic Antigen (10 mg or $20 \mathrm{mg}$ ), Allied Monitor, Missouri, United States).

Negative- and positive control antisera (Control antisera), Allied Monitor, Missouri, United States) were included in all tests. The antigen was placed in the central well and control and test samples in the outside wells. Test samples showing a precipitation line of identity with the antigen were reported as positive.

\section{PCR to detect MAP in feces}

The DNA from fecal specimens was extracted using a commercial DNA-extraction protocol, as per the manufacturer's instructions (Qiagen tissue DNA extraction protocol, California, United States). PCR to detect MAP in feces targeting ISMAP02 [12] was performed using a commercial kit (AgPath-ID ${ }^{\text {TM MAP }}$ (Johne's) Reagent Kit, Life Technologies/Albion, New York, United States) with an ABI7900 Real-Time Thermocycler in duplicate, according to manufacturer's recommendations. Both Cycle Threshold (CT) values and positive/negative results were obtained. CT values $\leq 37$ were considered a positive result and inconclusive samples were repeated.

\section{Statistical Analysis}

Sensitivity (Se) and specificity (Sp) were calculated for each assay compared to the known infection status at necropsy.
The sensitivity and specificity with 95\% Clopper-Pearson's Confidence Intervals [13] were estimated based on the $2 \times 2$ table for the comparison of the test kit with the reference test (infection status at necropsy). The sensitivity of all four tests was compared using the McNemar Test, defining 0.05 as the level of significance. $P$ values for the all pair-wise comparisons of sensitivity between four tests are adjusted for each time point using the Stepdown Bonferroni method of Holm (1979) [14].

\section{Results}

\section{Clinical signs}

Distinct clinical signs typically associated with JD were not observed in any challenged kid during the first 11 months postchallenge. In the last few weeks of the study, three kids from the experimental vaccine groups showed mild clinical signs consistent with JD, such as weight loss, decreased body condition scores, rough hair coats and pasty, non-pelleted feces. None of the kids from the positive control group (sham-vaccinated and challenged) or the commercial vaccine group showed clinical signs of JD.

\section{Goat serum samples}

Eighty serum samples were obtained from goats each month from 10 to 13 months post challenge, and were tested using the new ELISA, the approved ELISA and AGID assay. For all tests, the results from the 10 to 13 month post-challenge time point were analyzed for specificity and sensitivity and compared to the confirmed infection status at necropsy.

The results for the new ELISA are shown in Table 2. At 13 months, sensitivity was $55.7 \%$ and specificity was $100 \%$. At 13 months, sensitivity of the approved ELISA (Table 3) was 52.9\% and specificity was $100 \%$. For the AGID test (Table 4), sensitivity was $24.3 \%$ and specificity was $100 \%$.

\section{Goat fecal samples}

Eighty fecal samples were obtained from goats each month from 10 to 13 months post-challenge, and were tested for MAP using the PCR test kit. The PCR for MAP tests at 13 month postchallenge had a sensitivity of $87.1 \%$ and a specificity of $100 \%$ (Table 5).

\section{Sensitivity and specificity of the tests over time}

The percentage sensitivity of all four tests for samples taken from the last four months (months 10 to 13 post challenge) is shown in Figure 1. The specificity of all tests was $100 \%$ at all these time points. The sensitivity of all the tests increased over this period of time. Comparisons between the fecal PCR test and the (approved and new) ELISA tests, between the fecal PCR test and the AGID test, between the (approved and new) ELISA tests and the AGID test are statistically significant after Stepdown Bonferroni adjustment of the p-values at each time point. More details on p-values are shown in Table 6.

\section{Discussion}

For all the tests on serum samples from 10 to 13 months postchallenge, specificity was $100 \%$, which showed that a positive 
Table 2: Results for the new ELISA- Goat serum samples 10 to 13 months post-challenge.

\begin{tabular}{|c|c|c|}
\hline \multirow[b]{2}{*}{ At 13 months } & \multicolumn{2}{|c|}{ Confirmed Infection Status at Necropsy } \\
\hline & positive & negative \\
\hline New ELISA-positive & 39 (ТP) & 0 (FP) \\
\hline \multirow[t]{2}{*}{ New ELISA-negative } & $31(\mathrm{FN})$ & $10(\mathrm{TN})$ \\
\hline & Sensitivity & Specificity \\
\hline 13 months & $55.7 \%$ (95\%CI 43.3-67.6\%) & $100 \%$ (95\%CI 69.2-100\%) \\
\hline 12 months & $52.9 \%$ (95\%CI 40.6-64.9\%) & $100 \%(95 \%$ CI $69.2-100 \%)$ \\
\hline 11 months & $48.6 \%$ (95\%CI 36.4-60.8\%) & $100 \%(95 \%$ CI 69.2-100\%) \\
\hline 10 months & $40.0 \%$ (95\%CI $28.5-52.4 \%)$ & $100 \%$ (95\%CI 69.2-100\%) \\
\hline
\end{tabular}

Table 3: Results for the approved ELISA- Goat serum samples 10 to 13 months post-challenge.

\begin{tabular}{|c|c|c|}
\hline \multirow[b]{2}{*}{ At 13 months } & \multicolumn{2}{|c|}{ Confirmed Infection Status at Necropsy } \\
\hline & positive & negative \\
\hline Approved ELISA-positive & 37 (TP) & 0 (FP) \\
\hline \multirow[t]{2}{*}{ Approved ELISA-negative } & 33 (FN) & $10(\mathrm{TN})$ \\
\hline & Sensitivity & Specificity \\
\hline 13 months & 52.9\% (95\%CI 40.6-64.9\%) & $100 \%$ (95\%CI 69.2-100\%) \\
\hline 12 months & 44.3\% (95\%CI 32.4-56.7\%) & $100 \%$ (95\%CI 69.2-100\%) \\
\hline 11 months & 38.6\% (95\%CI 27.2-51.0\%) & $100 \%$ (95\%CI 69.2-100\%) \\
\hline 10 months & 35.7\% (95\%CI 24.6-48.1\%) & $100 \%(95 \% \mathrm{CI} 69.2-100 \%)$ \\
\hline
\end{tabular}

Table 4: Results for the AGID test.

\begin{tabular}{|c|c|c|}
\hline \multicolumn{3}{|c|}{ Confirmed Infection Status at Necropsy } \\
\hline At 13 months & positive & negative \\
\hline AGID-positive & 17 (TP) & 0 (FP) \\
\hline \multirow[t]{2}{*}{ AGID-negative } & $53(\mathrm{FN})$ & $10(\mathrm{TN})$ \\
\hline & Sensitivity & Specificity \\
\hline 13 months & $24.3 \%$ (95\%CI 14.8-36.0\%) & $100 \%$ (95\%CI 69.2-100\%) \\
\hline 12 months & 18.6\% (95\%CI 10.3-29.7\%) & $100 \%$ (95\%CI 69.2-100\%) \\
\hline 11 months & $10.0 \%$ (95\%CI 4.1-19.5\%) & $100 \%$ (95\%CI 69.2-100\%) \\
\hline 10 months & $24.3 \%$ (95\%CI 1.6-14.0\%) & $100 \%$ (95\%CI 69.2-100\%) \\
\hline
\end{tabular}

Table 5: Results for PCR test.

\section{Confirmed Infection Status at Necropsy}

\begin{tabular}{|l|l|l|}
\hline At 13 months & positive & negative \\
\hline PCR-positive & $\mathbf{6 1}(\mathrm{TP})$ & $\mathbf{0}(\mathrm{FP})$ \\
\hline PCR-negative & $\mathbf{9}(\mathrm{FN})$ & $\mathbf{1 0}(\mathrm{TN})$ \\
\hline & Sensitivity & Specificity \\
\hline $\mathbf{1 3}$ months & $87.1 \%(95 \%$ CI $77.0-93.9 \%)$ & $100 \%$ (95\%CI 69.2-100\%) \\
\hline $\mathbf{1 2}$ months & $80.0 \%(95 \%$ CI 68.7-88.6\%) & $100 \%$ (95\%CI 69.2-100\%) \\
\hline $\mathbf{1 1}$ months & $78.6 \%(95 \%$ CI 67.1-87.5\%) & $100 \%$ (95\%CI 69.2-100\%) \\
\hline $\mathbf{1 0}$ months & $65.7 \%(95 \%$ CI 53.4-76.7\%) & $100 \%$ (95\%CI 69.2-100\%) \\
\hline
\end{tabular}

TP=True Positive; FP=False Positive; FN=False Negative; TN=True Negative; CI=Confidence Interval 


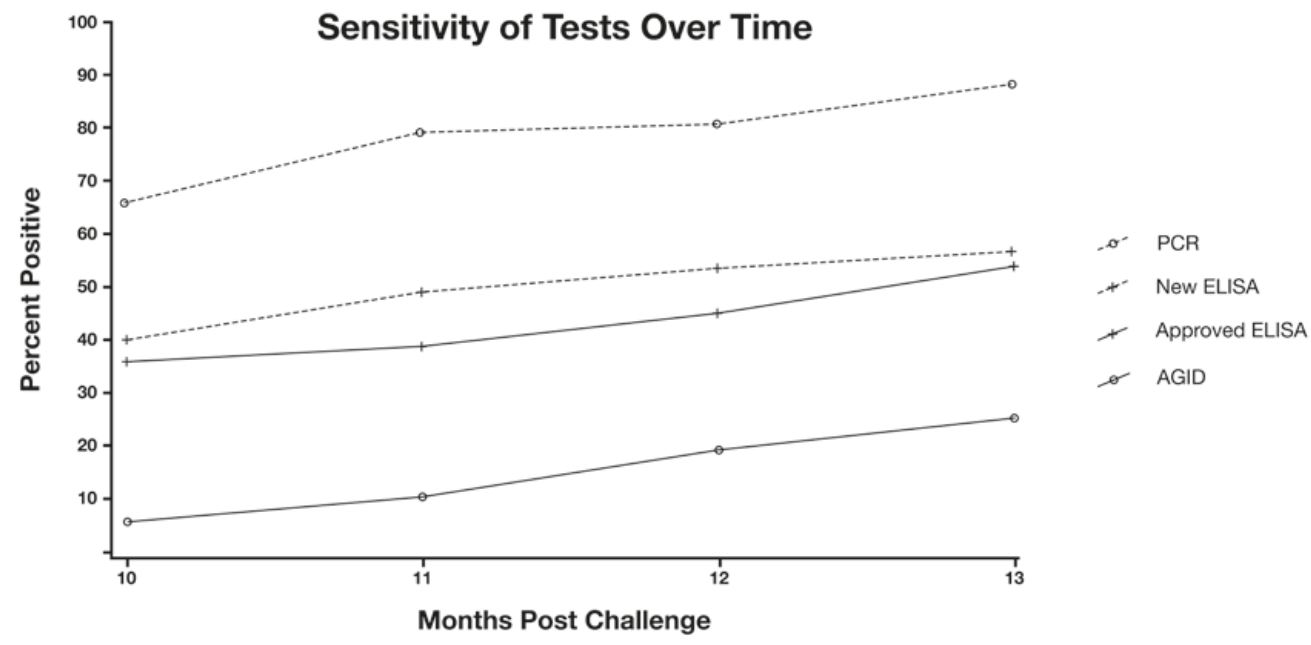

Figure 1: Sensitivity of the new ELISA approved ELISA, AGID and fecal PCR tests over time from 10 to 13 months post-challenge. For all four tests, sensitivity increased over time and specificity was $100 \%$ at all time points.

Table 6: Sensitivity comparison of all 4 tests 10 to 13 months post-challenge using the McNemar tests.

\begin{tabular}{|l|l|l|l|l|}
\hline Test comparison & Month 10 -value* & Month 11 $\boldsymbol{p}$-value* & Month 12 $\boldsymbol{p}$-value* & Month 13 $\boldsymbol{p}$-value \\
\hline Approved ELISA vs. AGID & $<0.0001$ & $<0.0001$ & 0.0002 & $<0.0001$ \\
\hline Fecal PCR vs. AGID & $<0.0001$ & $<0.0001$ & $<0.0001$ & $<0.0001$ \\
\hline Fecal PCR vs. approved ELISA & 0.0002 & $<0,0001$ & $<0.0001$ & $<0.0001$ \\
\hline Fecal PCR vs. new ELISA & 0.0013 & 0.0002 & 0.0013 & 0.0002 \\
\hline New ELISA vs. AGID & $<0.0001$ & $<0.0001$ & $<0.0001$ & $<0.0001$ \\
\hline New ELISA vs. approved ELISA & 0.2568 & 0.0196 & 0.0833 & 0.5637 \\
\hline "Raw $p$-values of McNemar tests are adjusted using Step-down Bonferroni procedure at each time point.
\end{tabular}

test result reliably indicated the presence of JD antibodies. For the 13 month samples, sensitivity of the serum antibody tests was $57.7 \%, 52.9 \%$ and $24.3 \%$ for the new ELISA, approved ELISA, and the AGID, respectively. The PCR test sensitivity for the fecal samples was $87.1 \%$. For the 11 month samples, sensitivities were $48.6 \%, 38.6 \%, 10.0 \%$ and $78.6 \%$ for the new ELISA, approved ELISA, and the AGID and the PCR test. These results were expected, given that PCR detects MAP DNA and is more sensitive than most serological methods for detection of MAP antibodies. However, for reasons of cost, ease of use and the rapid availability of results, ELISA tests are the preferred method of testing in this field. The sensitivity of the new ELISA test was consistent with the approved ELISA over most time points, but showed improved sensitivity, which was statistically-significant for the 11 month samples ( $p=0.0196)$.

The results for the new ELISA in goat serum are comparable with those reported when the ELISA test is used for cattle serum, but with a reduced level of sensitivity in goat serum compared to cattle serum [15]. Overall, the results demonstrate the ability of the new ELISA to effectively detect circulating MAP antibody in the serum of infected goats and this initial study indicates that the test has potential for use in goat JD control programs.
Obviously, further studies (of repeatability and reproducibility) should be conducted to validate its use in JD control programs.

A veterinarian in practice needs to know how to use the new ELISA test in goats as part of a JD control program and understand what the results mean. The results of this study confirm that, as is the case for all available JD diagnostic tests identifying MAP antibody, both ELISA tests have low sensitivity in the subclinical phase of JD, because antibodies are only consistently present in animals with more advanced disease showing clinical signs. In this study, clinical signs compatible with JD were not observed in any kid during the first 11 months post-challenge and even after that time were only seen in a very small minority of kids - three out of the 70 that were challenged.

Sensitivity of the new ELISA test increased over time postchallenge. In the 10 month samples, sensitivity was $40.0 \%$ and this increased in the 13 months samples to $55.7 \%$. Based on these findings, it is recommended that ELISA testing for JD should be done at the herd-level rather than individual-level, because in an infected herd, there are likely to be individuals in more advanced stages of disease that have MAP-antibodies. When testing at herd-level is sufficient, animals should be sampled to increase the probability of detecting at least one animal as positive in the herd. 
Calculation of the number of animals required for testing must take into consideration the JD prevalence rate (within the herd and amongst herds), herd cut-off value (i.e. number of positive animals that define a positive test result) and the number of animals in the herd [16].

The specificity of the new ELISA was $100 \%$, so a positive result in any goat strongly suggests that JD is present in the herd. If all ELISA results are negative, but there is a very high suspicion of JD in the herd, the fecal PCR test for JD should be used for follow-up testing.

If there is a need to use the ELISA test on individual goats, it is recommended that they should be at least one year old and show clinical signs compatible with JD.

In this study, there was no experimental group, in which kids were vaccinated but not challenged, so it was not possible to evaluate whether the tests would have given positive results as result of vaccine-induced antibodies alone. In a previous JD vaccine efficacy study [11], some kids were vaccinated with JD vaccines, but were not challenged. Testing for MAP-antibodies was conducted using the same approved ELISA and AGID tests as used in this study. Serum from some of the unchallenged, JD vaccinated kids tested positive for MAP-antibodies using either the ELISA or the AGID test, but curiously, no sample tested positive for both tests. The previous study [11] included some JD vaccines with QS21 adjuvant, designed to enhance cell-mediated immunity (Th-1 response) and others with alum adjuvant that preferentially induces a Th-2 response (antibody-mediated). In theory, vaccines designed to stimulate a Th-1 (cell-mediated) response should result in less antibody production, and should therefore, be less likely to give positive results in tests for MAPantibodies. However, the effects were not as strongly polarized as the authors had hoped, and some of the unchallenged JD kids given vaccines containing QS21 adjuvant tested positive for MAP-antibodies. Vaccination also affected the time course of development of detectable antibodies in kids that were challenged; time to first positive AGID and ELISA test results in JD vaccinated kids was reduced by approximately two months and post-challenge ELISA sensitivity was reduced compared to a group of kids sham-vaccinated with vaccine containing alum adjuvant and challenged [11].

It is clear that JD vaccination can result in the production of antibodies that cannot be differentiated from those produced by JD infection, and may alter the time, at which antibodies can first be detected. Both factors need to be taken into account when interpreting ELISA test results in vaccinated goats. An ELISA positive result in a vaccinated goat should be confirmed using the JD PCR test on fecal samples.

\section{Acknowledgements}

The authors gratefully thank the employees at the University of Georgia's Tifton Veterinary Diagnostic and Investigational Laboratory for their assistance on this project.

\section{Conflicts of Interest}

The study was funded by Zoetis, Inc. Ron White, Chinta
Lamichhane, Keith Marotti and Dan Lin is employed by Zoetis, Inc.

\section{References}

1. Clarke CJ. The pathology and pathogenesis of paratuberculosis in ruminants and other species. J Comp Pathol. 1997;116(3):217-61.

2. Stehman SM. Paratuberculosis in small ruminants, deer, and South American camelids. Vet Clin North Am Food Anim Pract. 1996;12(2):441-55.

3. Lovell R, Levi M, Francis J. Studies on the survival of Johne's bacilli. J. Comp Pathol. 1944;54:120-129.

4. Eamens GJ. Johne's disease in goats. In: Milner AR and Wood PR editors. Johne's disease, current trends in research, diagnosis and management. Commonwealth Scientific and Industrial Research Organization: East Melbourne, Australia; 1989. p. 105-112.

5. Merck Veterinary Manual. ed. Aiello SE. 8th edition. Whitehouse Station. New Jersey, United States: Merck and Co. Inc; 1998. p. $537-$ 538.

6. Benedictus G, Dujkhuizen AA, Stelwagen J. Economic losses due to paratuberculosis in dairy cattle. Vet Rec. 1987;121(7):142-6.

7. Lombard JE, Garry FB, McClusky BJ, Wagner, BA. Risk of removal and effects on milk production associated with paratuberculosis status in dairy cows. J Am Vet Med Assoc. 2005;227(12):1975-81.

8. Bull JT. MAP disease in humans: An inconvenient truth or a trivial dalliance. In: Proceedings of the 12th International Colloquium on Paratuberculosis. Parma, Italy; 2014.p. 307-8.

9. Munjal SK, Tripathi BN, Paliwal OP. Progressive immunopathological changes during early stages of experimental Infection of goats with Mycobacterium avium subspecies paratuberculosis. Vet Pathol. 2005;42:427-436.

10. Hines ME 2nd, Turnquist SE, Ilha MR, Rajeev S, Jones AL, Whittington $\mathrm{L}$, et al. Evaluation of novel oral vaccine candidates and validation of a caprine model of Johne's disease. Front Cell Infect Microbiol. 2014;4:26. doi: 10.3389/fcimb.2014.00026.

11. Hines ME, Stiver S, Giri D, Whittington L, Watson C, Johnson J, et al. Efficacy of spheroplastic and cell-wall competent vaccines for Mycobacterium avium subsp. paratuberculosis in experimentallychallenged baby goats. Vet Microbiol. 2007;120:261-283.

12. Stabel JR, Bannantine JP. Development of a nested PCR method targeting a unique multicopy element, ISMap02, for detection of Mycobacterium avium subsp. paratuberculosis in fecal samples. J. Clin. Microbiol. 2005;43:4744-4750.

13. Clopper CJ, Pearson ES. The use of confidence or fiducial limits illustrated in the case of the binomial. Biometrika. 1934;26:404-413.

14. Holm, S. A simple sequentially rejective multiple test procedure. Scandinavian Journal of Statistics. 1979;6:65-70.

15.Zoetis Data on File. USDA Submission Report, May 14, 2014.

16. Thrusfield M. Veterinary Epidemiology. 1st ed. ButterworthHeinemann, editors. Oxford, UK; 1986. 